MATHEMATICS OF COMPUTATION

Volume 75, Number 256, October 2006, Pages 1735-1753

S 0025-5718(06)01875-8

Article electronically published on May 23, 2006

\title{
ORDER OF CONVERGENCE OF SECOND ORDER SCHEMES BASED ON THE MINMOD LIMITER
}

\author{
BOJAN POPOV AND OGNIAN TRIFONOV
}

\begin{abstract}
Many second order accurate nonoscillatory schemes are based on the minmod limiter, e.g., the Nessyahu-Tadmor scheme. It is well known that the $L_{p}$-error of monotone finite difference methods for the linear advection equation is of order $1 / 2$ for initial data in $W^{1}\left(L_{p}\right), 1 \leq p \leq \infty$. For second or higher order nonoscillatory schemes very little is known because they are nonlinear even for the simple advection equation. In this paper, in the case of a linear advection equation with monotone initial data, it is shown that the order of the $L_{2}$-error for a class of second order schemes based on the minmod limiter is of order at least $5 / 8$ in contrast to the $1 / 2$ order for any formally first order scheme.
\end{abstract}

\section{INTRODUCTION}

We are interested in the scalar hyperbolic conservation law

$$
\begin{cases}u_{t}+f(u)_{x}=0, & (x, t) \in \mathbb{R} \times(0, \infty), \\ u(x, 0)=u^{0}(x), & x \in \mathbb{R},\end{cases}
$$

where $f$ is a given flux function. In recent years, there has been enormous activity in the development of the mathematical theory and in the construction of numerical methods for (11). Even though the existence-uniqueness theory of weak solutions is complete, there are many numerically efficient methods for which the questions of convergence and error estimates are still open. For example, there are many nonoscillatory schemes based on a variety of nonlinear limiters which are numerically robust, at least in many numerical tests, but theoretical results about convergence and error estimates are still missing [1, 5, 6, 16. In the case of linear flux, there is a unique solution of (1). Therefore, a total variation diminishing (TVD) property guarantees convergence of such schemes; however, the question regarding the accuracy of these schemes still remains open.

In this paper, we consider a class of the so-called Godunov-type schemes for solving (1). There are two main steps in such schemes: evolution and projection. In the original Godunov scheme, the projection is onto a piecewise constant function such that its value in each cell is the cell average of the approximate solution after the evolution step. In the general Godunov-type method, the projection is onto a

Received by the editor April 22, 2004 and, in revised form, July 6, 2005.

2000 Mathematics Subject Classification. Primary 65M15; Secondary 65M12.

Key words and phrases. Conservation laws, error estimates, second order schemes, minmod limiter.

The first author was supported in part by NSF DMS Grant \#0510650.

The second author was supported in part by NSF DMS Grant \#9970455.

(C)2006 American Mathematical Society Reverts to public domain 28 years from publication 
piecewise polynomial function. To determine the properties of these schemes it is necessary to study the properties of the projection operator. We limit our attention to second order Godunov-type schemes based on the minmod limiter. Namely, the piecewise linear projection in such schemes is based on cell averages and uses the minmod limiter for the slope reconstruction (see (4) and (5) in the next section). We call such a scheme minmod-type. For example, the Nessyahu-Tadmor (NT) scheme 14 is of minmod-type and it is based on staggered evolution - we alternate between two staggered grids (see Section 2 or 14 for details). Other examples include the second order nonoscillatory central schemes with nonstaggered grids given in [7, the TVD2 scheme in [5], and the high resolution TVD schemes in [4, 17]. Theoretical results about convergence of such schemes to the entropy solution, or error estimates, are still missing. In most cases, the authors give a variation bound for such a scheme which is enough to conclude that the method converges to a weak solution (see [10]). In the case of linear flux, the conservation law (11) is the usual linear advection equation. The theory of linear numerical schemes for the linear advection equation is well developed and is presented in detail in [2. It is shown that if a linear finite difference scheme is of formal order $\mu$ and $L_{2}$-stable, then the order of convergence in $L_{2}$ is exactly $\frac{s \mu}{\mu+1}$ for initial data in $W^{s}\left(L_{2}\right)$. The case $1 \leq p \leq \infty, p \neq 2$ is also analyzed but the theory is more complicated (see [2] for details). Hence, the order of convergence increases with the formal order of the linear scheme. In the case of BV $(\mathbb{R})$ initial data, Tang and Teng show in 18 that all monotone schemes (a special case of first order schemes used in conservation laws) are exactly $1 / 2$ order accurate in $L_{1}$. This result was later extended to the nonlinear case in [15]. Therefore, these schemes cannot be very accurate in computations. From a numerical point of view, the formal second or higher order nonoscillatory schemes developed in [4, 17, 5, 6, 14, 8, 13] are much better than any monotone scheme, but theoretically there was no rigorous result confirming the numerical evidence even in the case of a linear advection equation. The reason is that many high order schemes used in conservation laws are based on limiters and are nonlinear even in the simplest case of linear advection. Therefore, the results in [2] are not valid for any nonoscillatory scheme based on limiters. The only known result was that a nonoscillatory total variation bounded scheme is at least $1 / 2$ order accurate in $L_{1}$. Recently in [11, we showed that the second order schemes based on the minmod limiter (NT or TVD2 for example) are at least $1 / 2$ order accurate in $L_{2}$ for any initial data in $W^{1}\left(L_{2}(\mathbb{R})\right)$. In both cases, the order of convergence proven for the second order schemes is the same as the order for the first order (for example monotone) schemes. In general, it was not clear how to prove better error estimates for second or higher order nonoscillatory or simply nonlinear schemes even in the case of a scalar linear advection equation. The difficulty in proving better estimates is that even though the minmod-type schemes are formally second order, they are known to preserve only first order smoothness of the initial data:

(i) the total variation for initial data in $\mathrm{BV}(\mathbb{R})$;

(ii) a discrete $\left(l_{2}\right)$ norm of the first derivative for initial data in $W^{1}\left(L_{2}(\mathbb{R})\right)$ (see [11]).

Hence, all existing error estimates were proven in the same way as for monotone schemes because they also preserve the above functionals. Here, we develop a new approach for proving better error estimates for second order schemes in the case of the linear advection equation with monotone initial data in $W_{\text {loc }}^{1}\left(L_{2}(\mathbb{R})\right)$. 
Namely, we measure how well a scheme approximates a discrete $\left(l_{2}\right)$ norm of the first derivative in time. In our earlier paper [11, we showed that some first (Godunov and $\mathrm{LxF}$ ) and second order (NT and TVD2) schemes diminish the $l_{2}$-norm of the first derivative in time and based on that we derived an error estimate with a rate $1 / 2$ for any initial data in $W^{1}\left(L_{2}(\mathbb{R})\right)$. Here, we prove that this decay for a minmod-type second order scheme is different (smaller) than the decay of any first order scheme in the case of a monotone initial data. The new decay estimate allows us show that the error of the standard nonoscillatory second order scheme is at least $5 / 8$ for any monotone initial data $u^{0} \in W_{\text {loc }}^{1}\left(L_{2}(\mathbb{R})\right)$ with $\frac{d u^{0}}{d x} \in L_{2}(\mathbb{R})$. The restriction to monotone initial data is natural because a standard minmodtype scheme reduces to a formal first order scheme at any local extremum. In the paper we refer to the upwind scheme as TVD2 because the other two upwind minmod-type schemes [4, 17] coincide with the TVD2 scheme for linear flux.

The paper is organized as follows. In Section 2, we describe the generic second order minmod-type schemes: the staggered minmod scheme (NT) and the upwind minmod scheme in the case of a linear advection equation. Then, we give a new decay estimate (see Lemma 1) for a discrete norm of the first derivative of the numerical solution in time. In Section 3, we present our main result: minmod-type schemes have a better convergence rate than first order schemes for monotone initial data. In the Appendix, we give the proof of Lemma 1. The results in this paper and our previous publication [11] are important steps in obtaining convergence results and error estimates for second order nonoscillatory schemes in the case of a conservation law with strictly convex flux.

\section{StABILITY OF NONOSCILLATORY MINMOD-TYPE SCHEMES}

In this section, we are concerned with nonoscillatory difference approximations to the linear advection equation

$$
\begin{cases}u_{t}+a u_{x}=0, & (x, t) \in \mathbb{R} \times(0, \infty) \\ u(x, 0)=u^{0}(x), & x \in \mathbb{R}\end{cases}
$$

The prototypes of all high order nonoscillatory schemes are the second order schemes based on a piecewise linear spacial reconstruction. We restrict our attention to the standard representatives: the usual TVD2 scheme (see, e.g., [5], and the staggered Nessyahu-Tadmor (NT) scheme [14). In the case of a linear flux, $f(u)=a u$, and uniform space and time meshes, both schemes reduce to the same type of iterative relation between the sequences of new and old cell averages (note that the NT scheme alternates between two uniform grids). For simplicity, we only consider the case $a \geq 0$ with the other case being analogous. We are going to show that the relationship between new and old cell averages in any of the two schemes (TVD2 or NT) is

$$
w_{j}^{\prime}=\alpha w_{j-1}+(1-\alpha) w_{j}+\frac{1}{2} \alpha(1-\alpha)\left(\mathrm{m}\left(\delta_{j-1}, \delta_{j}\right)-\mathrm{m}\left(\delta_{j}, \delta_{j+1}\right)\right),
$$

where $\mathrm{m}(a, b)$ stands for the minmod limiter

$$
\mathrm{m}(a, b) \equiv \operatorname{MinMod}(a, b):=\frac{1}{2}(\operatorname{sgn}(a)+\operatorname{sgn}(b)) \cdot \min (|a|,|b|),
$$

and $\delta_{j}:=w_{j}-w_{j-1}, j \in \mathbb{Z}$. We use $\left\{w_{j}\right\}$ for the sequence of cell averages of the numerical solution $v$ at the old time step $t_{n}=n \Delta t$ and $\left\{w_{j}^{\prime}\right\}$ for the sequence of cell averages of the numerical solution $v$ at the new time step $t_{n+1}=(n+1) \Delta t$. 
We start with the upwind scheme TVD2. Let $x_{j}:=j \Delta x$ and $\lambda:=\frac{\Delta t}{\Delta x}$. We denote the numerical solution at time step $t_{n}$ with $v^{n}, v^{n}:=v\left(\cdot, t_{n}\right)$, and its cell averages on each cell $I_{j}:=\left(x_{j-1 / 2}, x_{j+1 / 2}\right)$ with $v_{j}^{n}, j \in \mathbb{Z}$. Note that the cell averages of $v^{0}$ are equal to the cell averages of the initial condition $u^{0}: v_{j}^{0}:=u_{j}^{0}$, $j \in \mathbb{Z}$. The numerical solution $v\left(\cdot, t_{n}\right)$ is a linear function on each cell $I_{j}$ given by the minmod reconstruction

$$
\left.v^{n}\right|_{I_{j}}=v_{j}^{n}+\left(x-x_{j}\right) \frac{1}{\Delta x} \mathrm{~m}\left(v_{j+1}^{n}-v_{j}^{n}, v_{j}^{n}-v_{j-1}^{n}\right) .
$$

The cell averages $v_{j}^{n+1}$ of the numerical solution at the next time step are determined by averaging the exact solution of (2) with initial condition $v^{n}$ at time $\Delta t$. The new averages $v_{j}^{n+1}$, can be written in terms of the old averages $v_{j}^{n}$ and the old jumps $\delta_{j}^{n}:=v_{j}^{n}-v_{j-1}^{n}, j \in \mathbb{Z}$, in the following way (see section 7 of [5]):

$$
v_{j}^{n+1}=\lambda a v_{j-1}^{n}+(1-\lambda a) v_{j}^{n}+\frac{\lambda a}{2}(1-\lambda a)\left(\mathrm{m}\left(\delta_{j-1}^{n}, \delta_{j}^{n}\right)-\mathrm{m}\left(\delta_{j}^{n}, \delta_{j+1}^{n}\right)\right)
$$

for $n \geq 0$. The CFL condition in this case is $0 \leq \lambda a \leq 1$. Note that the formula above is (3) with $\alpha=\lambda a$.

In the case of the NT scheme, we alternate between the cell averages on two staggered uniform partitions (see [14]). In order to formulate the iterative relation in the same way as in the upwind case, we define $x_{j}:=j \Delta x, I_{j}^{n}:=$ $\left(x_{j}+\frac{(n-1) \Delta x}{2}, x_{j}+\frac{(n+1) \Delta x}{2}\right)$, and $\alpha:=\frac{1}{2}+\lambda a$. Similar to the TVD2 case, the numerical solution $v^{n}:=v\left(\cdot, t_{n}\right)$ is determined from its cell averages by the minmod reconstruction

$$
\left.v^{n}\right|_{I_{j}^{n}}=v_{j}^{n}+\left(x-x_{j}-\frac{n \Delta x}{2}\right) \frac{1}{\Delta x} \mathrm{~m}\left(v_{j+1}^{n}-v_{j}^{n}, v_{j}^{n}-v_{j-1}^{n}\right), \quad j \in \mathbb{Z},
$$

where $v_{j}^{n}:=\frac{1}{\Delta x} \int_{I_{j}^{n}} v(x, t) d x$. Then, the cell averages $v_{j}^{n+1}$ at the next time step are determined by averaging the exact solution of (2) with initial condition $v^{n}$ at time $\Delta t$. The new averages $v_{j}^{n+1}$ can be written using the iterative formula (3) with $\alpha:=\frac{1}{2}+\lambda a$ (see [11]) and the CFL condition in this case is $0 \leq \frac{1}{2}+\lambda a=\alpha \leq 1$. The difference from the upwind case is that

(i) When $n$ is even, the sequence of cell averages $\left\{v_{j}^{n}\right\}$ represents the averages of the numerical solution on the regular partition $I_{j}=\left(x_{j}-\frac{\Delta x}{2}, x_{j}+\frac{\Delta x}{2}\right), j \in \mathbb{Z}$;

(ii) When $n$ is odd, the sequence of cell averages $\left\{v_{j}^{n}\right\}$ represents the averages of the numerical solution on the staggered partition $J_{j}:=\left(x_{j}, x_{j+1}\right), j \in \mathbb{Z}$.

In both cases, one can define a global numerical solution $v$ corresponding to the initial data $u^{0}$ in the following way. Let $v(x, t)$ be a right-continuous function in $t$ such that, for each $n=0, \ldots, N-1, v$ is the solution of

$$
\begin{cases}u_{t}^{n}+a u_{x}^{n}=0, & (x, t) \in \mathbb{R} \times\left(t_{n}, t_{n+1}\right), \\ u(x, 0)=v^{n}(x), & x \in \mathbb{R}\end{cases}
$$

Note that $v$ is uniquely determined by the functions $\left\{v^{n}\right\}_{n=0}^{N-1}$, where $v^{n}$ is the minmod piecewise linear reconstruction defined in (5) from the cell averages of $v\left(\cdot, t_{n}^{-}\right)$with $v\left(\cdot, 0^{-}\right):=u^{0}$. In the TVD2 case, we have the same uniform grid for each step, and in the NT case we alternate between two staggered uniform grids. In both cases, we end up with the same type of iterative relation between the cell averages of the numerical solution (see (3)), just the value of $\alpha$ is different. 
We now present a new stability property for a numerical method which satisfies (8). Namely, we assume that the new averages $v_{j}^{n+1}:=w_{j}^{\prime}$ can be written in terms of the old averages $v_{j}^{n}:=w_{j}$ and the old jumps $\delta_{j}^{n}:=v_{j}^{n}-v_{j-1}^{n}$ by (3). The CFL condition is $0 \leq \alpha \leq 1$ which covers both schemes (TVD2 and NT). Let us recall the iterative formula (3) with the time index in place:

$$
v_{j}^{n+1}=\alpha v_{j-1}^{n}+(1-\alpha) v_{j}^{n}+\frac{1}{2} \alpha(1-\alpha)\left(\mathrm{m}\left(\delta_{j-1}^{n}, \delta_{j}^{n}\right)-\mathrm{m}\left(\delta_{j}^{n}, \delta_{j+1}^{n}\right)\right) .
$$

Using (9), we derive the formula for the sequence of new jumps $\left\{\delta_{j}^{n+1}\right\}$ in terms of the old ones $\left\{\delta_{j}^{n}\right\}$ :

$$
\delta_{j}^{n+1}=\alpha \delta_{j-1}^{n}+(1-\alpha) \delta_{j}^{n}-\beta \mathrm{m}\left(\delta_{j-2}^{n}, \delta_{j-1}^{n}\right)+2 \beta \mathrm{m}\left(\delta_{j-1}^{n}, \delta_{j}^{n}\right)-\beta \mathrm{m}\left(\delta_{j}^{n}, \delta_{j+1}^{n}\right),
$$

where $\beta:=\frac{1}{2} \alpha(1-\alpha)$.

In our previous paper [11, we proved that the the iterative formula (10) does not increase the $l_{2}$-norm of the jumps of the numerical solution in time. That is,

$$
\sum_{j}\left(\delta_{j}^{n+1}\right)^{2}-\sum_{j}\left(\delta_{j}^{n}\right)^{2} \leq 0
$$

for all $n=0,1, \ldots$ The above stability result can be easily verified for any monotone scheme because in that case the new cell averages are a convex combination of the old ones. For a first order scheme (including all monotone schemes) the difference $\sum_{j}\left(\delta_{j}^{n}\right)^{2}-\sum_{j}\left(\delta_{j}^{n+1}\right)^{2}$ is of order $\sum_{j}\left(\delta_{j}^{n}-\delta_{j-1}^{n}\right)^{2}$. This reflects the diffusive nature of the first order approximation. For the exact solution, there is no decay in time of any shift invariant norm. But for a first order numerical solution we expect, and it is easy to show for the Godunov and LxF schemes, the following numerical viscosity in the decay of the first derivative $l_{2}$-norm:

$$
\sum_{j}\left(\delta_{j}^{n}\right)^{2}-\sum_{j}\left(\delta_{j}^{n+1}\right)^{2}=O\left(\sum_{j}\left(\delta_{j}^{n}-\delta_{j-1}^{n}\right)^{2}\right)=O\left(\sum_{j}\left(v_{j+1}^{n}-2 v_{j}^{n}+v_{j-1}^{n}\right)^{2}\right) .
$$

Here, we derive an improved stability estimate for the second order minmod-type schemes given in (9). Namely, we give an exact estimate for the time decay of the discrete $l_{2}$-norm in the case of a monotone initial condition. We claim

Lemma 1. Let $u^{0}$ be any monotone function and $\left\{v^{n}\right\}, n=0,1, \ldots$, be the sequence approximate solutions $v^{n}$ defined by (9). Then, we have

$$
3 \beta \sum_{j}\left(\Delta^{2} \delta_{j}^{n}\right)^{2} \geq \sum_{j}\left(\delta_{j}^{n}\right)^{2}-\sum_{j}\left(\delta_{j}^{n+1}\right)^{2} \geq \frac{\beta^{3}}{4} \sum_{j}\left(\Delta^{2} \delta_{j}^{n}\right)^{2},
$$

where $\beta=\frac{1}{2} \alpha(1-\alpha)$ and $\Delta^{2} \delta_{j}^{n}=\delta_{j}^{n}-2 \delta_{j-1}^{n}+\delta_{j-2}^{n}$.

The proof of this lemma is given in the Appendix. We want to point out the main difference: for a first order scheme, we have a decay for $\sum_{j}\left(\delta_{j}^{n}\right)^{2}-\sum_{j}\left(\delta_{j}^{n+1}\right)^{2}$ of order $\sum_{j}\left(v_{j+1}^{n}-2 v_{j}^{n}+v_{j-1}^{n}\right)^{2}$, and for a second order scheme we get a decay $\sum_{j}\left(v_{j+1}^{n}-3 v_{j}^{n}+3 v_{j-1}^{n}-v_{j-2}^{n}\right)^{2}$ by Lemma 1. We will use this decay estimate in the next section to derive our main result. 


\section{ERROR ESTIMATES FOR SECOND ORDER SCHEMES}

In this section we present our main result. Namely, we will show that minmodtype schemes have a better convergence rate than first order schemes for any monotone initial condition $u^{0} \in W_{\text {loc }}^{1}\left(L_{2}\right)$ with $\frac{d u^{0}}{d x} \in L_{2}(\mathbb{R})$. In the text below, we refer to a first order scheme as exactly first order, which means that it is not also a second or higher order scheme. Let us first recall some results for first order linear schemes (see Theorem 4.2 and Theorem 4.4 in [2]).

Theorem 2. Let $u^{0} \in W^{1}\left(L_{2}(\mathbb{R})\right), u(x, t)=u^{0}(x-a t)$ be the exact solution of (2), and $v$ be a numerical solution consistent with (2) and exactly first order accurate. If the space and time meshes are $h:=\Delta x$ and $\Delta t$ with $\Delta t / h=\lambda=$ const, then there exists a constant $C>0$ which depends on the final time $T>0$ such that

$$
\|u(\cdot, T)-v(\cdot, T)\|_{L_{2}} \leq C h^{1 / 2}\left\|u^{0}\right\|_{W^{1}\left(L_{2}(\mathbb{R})\right)} \cdot
$$

Moreover, the above estimates is optimal over the class $W^{1}\left(L_{2}(\mathbb{R})\right)$. That is, there exists a constant $c>0$ such that

$$
\sup \left\{\|u(\cdot, T)-v(\cdot, T)\|_{L_{2}}: u^{0} \in \hat{C}^{\infty},\left\|u^{0}\right\|_{W^{1}\left(L_{2}(\mathbb{R})\right)} \leq 1\right\} \geq c h^{1 / 2},
$$

where $\hat{C}^{\infty}$ is the set of all functions with Fourier transform in $C_{0}^{\infty}(\mathbb{R})$.

The above result is also valid for any monotone scheme except in the trivial case of pure translation which corresponds to $\alpha=0$ or $\alpha=1$ here (see Remark 1 in [18] and Section 3.3 in [2]). Using the linearity of the problem and the numerical scheme, it is easy to show

Corollary 3. The convergence rate for any linear exactly first order scheme is not better than $1 / 2$ for the class of all monotone initial conditions $u^{0} \in W_{\text {loc }}^{1}\left(L_{2}(\mathbb{R})\right)$ with $\frac{d u^{0}}{d x} \in L_{2}(\mathbb{R})$. That is, an estimate of the type

$$
\|u(\cdot, T)-v(\cdot, T)\|_{L_{2}} \leq C h^{\gamma}\left|u^{0}\right|_{W^{1}\left(L_{2}(\mathbb{R})\right)}
$$

for all monotone $u^{0}$ is not possible for $\gamma>1 / 2$.

The seminorm $\left|u^{0}\right|_{W^{1}\left(L_{2}(\mathbb{R})\right)}$ is a natural replacement of the full norm in estimates for equations with monotone initial conditions. It is also important to note that all results above, including the lower bound (13), hold for initial conditions $u^{0}$ which have first derivatives with compact support. That is, the error estimate and the lower bound in Theorem 2 do not depend on the infinite space domain. In the case of the Godunov or LxF schemes one can give an example of a monotone function with a derivative with compact support (similar to the construction in [18]) which realizes the lower bound (13). Hence, there are no "log" effects and the rate of convergence is exactly $1 / 2$ for these two schemes.

The following theorem is our main result. That is, the standard second order minmod-type schemes, NT and TVD2, have a better rate of convergence than the rate of any formal first order (including monotone) scheme.

Theorem 4. Let $u^{0} \in W_{\text {loc }}^{1}\left(L_{2}(\mathbb{R})\right)$ be monotone with $\frac{d u^{0}}{d x} \in L_{2}(\mathbb{R}), u(x, t)=$ $u^{0}(x-a t)$ be the exact solution of (2), and $v$ be the numerical solution generated by a standard minmod-type scheme (NT or TVD2) ( see (8)-(9)). If the CFL condition $0<\alpha<1$ is satisfied, $h:=\Delta x, t_{n}=n \Delta t, 0 \leq n \leq N$, and $T=N \Delta t$, we have

$$
\|u(\cdot, T)-v(\cdot, T)\|_{L^{2}} \leq C h^{5 / 8}\left|u^{0}\right|_{W^{1}\left(L_{2}(\mathbb{R})\right)},
$$


where $C$ is a constant which depends only on the final time $T$ and $\alpha$.

Proof. The error estimate is based on a refinement of the dual argument in [1] and the new stability result Lemma 1. In the proof, $C$ will be an absolute constant that can be different at different places. It is enough to prove the estimate for initial condition $u^{0}$ which has a derivative with compact support. The general result follows by standard arguments using the local dependence of the exact and the numerical solutions.

Let $e(x, t):=u(x, t)-v(x, t)$ be the difference between the global numerical solution $v$ defined in (8) and the exact solution $u$, and $E(x, t):=\int_{-\infty}^{x} e(s, t) d s$. Note that $u^{0}-v^{0}$ is zero for $x \in(-\infty, M)$, for some $M \in \mathbb{R}$. Therefore $E$ is well defined for all $(x, t) \in \mathbb{R} \times(0, T)$. We have that $E$ also satisfies (2) for $n \Delta t \leq t<(n+1) \Delta t$ with initial data $\int_{-\infty}^{x} u(s, n \Delta t)-v^{n}(s) d s, n=0,1, \ldots, N-1$. For a function $g \in L^{1}(\mathbb{R})$, we define a minus-one norm as

$$
\|g\|_{-1,2}:=\left\|\int_{-\infty}^{\cdot} g(s) d s\right\|_{L^{2}(\mathbb{R})} .
$$

It is easy to verify that for any $\tau \in \mathbb{R}$

$$
\left\|S_{\tau} g\right\|_{-1,2}=\|g\|_{-1,2},
$$

where $S_{\tau}$ is the shift operator $S_{\tau} g(\cdot):=g(\cdot-\tau)$.

Let us denote the piecewise linear minmod reconstruction (5) with $P_{h}$. That gives $v^{n}=P_{h} v\left(\cdot, t_{n}-\right)$ for $n=0,1, \ldots, N$, and $u(\cdot, t)=S_{t}\left(u^{0}\right)$ for any $t \geq 0$. Recall that $T=N \Delta t$. Then, we have the representations $u(T, \cdot)=\left(S_{a \Delta t}\right)^{N} u^{0}$ and $v(T, \cdot)=v^{N}=P_{h}\left(S_{a \Delta t} P_{h}\right)^{N} u^{0}$. We have

$$
\|e(\cdot, T)\|_{-1,2}=\left\|\left(S_{a \Delta t}\right)^{N} u^{0}-P_{h}\left(S_{a \Delta t} P_{h}\right)^{N} u^{0}\right\|_{-1,2},
$$

and by the triangle inequality we obtain

$$
\begin{aligned}
\|e(\cdot, T)\|_{-1,2} \leq & \left\|\left(S_{a \Delta t}\right)^{N} u^{0}-\left(S_{a \Delta t} P_{h}\right)^{N} u^{0}\right\|_{-1,2} \\
& +\left\|P_{h}\left(S_{a \Delta t}\right)^{N} u^{0}-\left(S_{a \Delta t} P_{h}\right)^{N} u^{0}\right\|_{-1,2} .
\end{aligned}
$$

Using (16) in (17), we obtain

$$
\|e(\cdot, T)\|_{-1,2} \leq\left\|\left(S_{a \Delta t}\right)^{N-1} u^{0}-P_{h}\left(S_{a \Delta t} P_{h}\right)^{N-1} u^{0}\right\|_{-1,2}+\left\|v^{N}-v\left(\cdot, t_{N}-\right)\right\|_{-1,2} .
$$

Let $e^{n}$ be the difference between the exact and the numerical solution at time $t_{n}=n \Delta t$. That is, $e_{n}:=\left(\left(S_{a \Delta t}\right)^{n}-P_{h}\left(S_{a \Delta t} P_{h}\right)^{n}\right) u^{0}, n=0,1, \ldots, N$. Then (18) is equivalent to

$$
\left\|e^{N}\right\|_{-1,2} \leq\left\|e^{N-1}\right\|_{-1,2}+\left\|P_{h} v\left(\cdot, t_{N}-\right)-v\left(\cdot, t_{N}-\right)\right\|_{-1,2},
$$

and applying (19) for $n=N, N-1, \ldots, 1$, we get

$$
\left\|e^{N}\right\|_{-1,2} \leq \sum_{n=0}^{N}\left\|P_{h} v\left(\cdot, t_{n}-\right)-v\left(\cdot, t_{n}-\right)\right\|_{-1,2} .
$$

We now estimate the minus-one norm of $P_{h} v\left(\cdot, t_{n}-\right)-v\left(\cdot, t_{n}-\right)$ in terms of the usual $L_{2}$-norm. In order to simplify the notation, we will use $v^{n,-}:=v\left(\cdot, t_{n}-\right)$, 
and only consider the upwind case (TVD2 scheme) with the staggered case (NT scheme) being analogous. We have

$$
\begin{aligned}
\left\|v^{n}-v^{n,-}\right\|_{-1,2}^{2} & =\int_{\mathbb{R}}\left(\int_{-\infty}^{x}\left(v^{n}(s)-v^{n,-}(s)\right) d s\right)^{2} d x \\
& =\sum_{j \in \mathbb{Z}} \int_{x_{j-1 / 2}}^{x_{j+1 / 2}}\left(\int_{x_{j-1 / 2}}^{x}\left(v^{n}-v^{n,-}\right)(s) d s\right)^{2} d x,
\end{aligned}
$$

where we use in (21) that $v^{n}=P_{h} v^{n,-}$ is a conservative approximation of $v^{n}$, i.e., $\int_{x_{j-1 / 2}}^{x_{j+1 / 2}}\left(v^{n}-v^{n,-}\right)(s) d s=0$ for all $j \in \mathbb{Z}$. Note that in the case of the NT scheme we need to use conservation over the staggered intervals $\left(x_{j}, x_{j+1}\right), j \in \mathbb{Z}$, for odd $n$. After applying the Cauchy-Schwarz inequality in (21), we obtain

$$
\begin{aligned}
\left\|v^{n}-v^{n,-}\right\|_{-1,2}^{2} & \leq \sum_{j \in \mathbb{Z}} \int_{x_{j-1 / 2}}^{x_{j+1 / 2}}\left(x-x_{j-1 / 2}\right) \int_{x_{j-1 / 2}}^{x}\left(v^{n}(s)-v^{n,-}(s)\right)^{2} d s d x \\
& \leq \sum_{j \in \mathbb{Z}} \int_{x_{j-1 / 2}}^{x_{j+1 / 2}}\left(x-x_{j-1 / 2}\right) d x \int_{x_{j-1 / 2}}^{x_{j+1 / 2}}\left(v^{n}(s)-v^{n,-}(s)\right)^{2} d s \\
& =\frac{h^{2}}{2}\left\|v^{n}-v^{n,-}\right\|_{L_{2}(\mathbb{R})}^{2} .
\end{aligned}
$$

Therefore, the error estimate (20) reduces to

$$
\left\|e^{N}\right\|_{-1,2} \leq \frac{h}{\sqrt{2}} \sum_{n=0}^{N}\left\|v^{n}-v^{n,-}\right\|_{L_{2}(\mathbb{R})} .
$$

We now estimate the term $\left\|v^{n}-v^{n,-}\right\|_{L_{2}(\mathbb{R})}$. Because the minmod operator $P_{h}$ is nonlinear, we will use an intermediate approximation $\tilde{P}_{h}$ defined as

$$
\left.\tilde{P}_{h} v^{n,-}\right|_{I_{j}}=v_{j}^{n}+\frac{x-x_{j}}{h}\left(v_{j+1}^{n}-v_{j}^{n}\right),
$$

for $j \in \mathbb{Z}$. It is a straightforward computation that

$$
\left\|P_{h} v^{n,-}-\tilde{P}_{h} v^{n,-}\right\|_{L_{2}(\mathbb{R})} \leq C h^{1 / 2}\left\|\Delta^{2} v_{j}^{n}\right\|_{l_{2}} .
$$

Hence, we have the estimate

$$
\left\|v^{n}-v^{n,-}\right\|_{L_{2}(\mathbb{R})} \leq C h^{1 / 2}\left\|\left\{\Delta^{2} v_{j}^{n}\right\}\right\|_{l_{2}}+\left\|\tilde{P}_{h} v^{n,-}-v^{n,-}\right\|_{L_{2}(\mathbb{R})} .
$$

$\tilde{P}_{h}$ is a linear operator based on local averages. The local approximation properties of $\tilde{P}_{h}$ were analyzed in [9]. Using that result, it is easy to show that

$$
\begin{aligned}
& \left\|\tilde{P}_{h} g-g\right\|_{L_{2}(\mathbb{R})} \leq C h^{2}\left|g^{\prime \prime}\right|_{L_{2}(\mathbb{R})}, \quad \text { for } g \in W^{2}\left(L_{2}(\mathbb{R})\right), \\
& \left\|\tilde{P}_{h} g\right\|_{L_{2}(\mathbb{R})} \leq C\|g\|_{L_{2}(\mathbb{R})} \quad \text { for } g \in L_{2}(\mathbb{R}) .
\end{aligned}
$$

Then, by standard approximation theory arguments (see Chapter 7 in [3]), we derive

$$
\left\|\tilde{P}_{h} g-g\right\|_{L_{2}(\mathbb{R})} \leq C \omega_{2}(g, h)_{L_{2}(\mathbb{R})}
$$

for any $g \in L_{2}(\mathbb{R})$. Here $\omega_{2}(g, h)_{L_{2}(\mathbb{R})}$ is the second modulus of smoothness of $g$ in $L_{2}$ :

$$
\omega_{2}(g, h)_{L_{2}(\mathbb{R})}:=\sup _{0 \leq s \leq h}\|g(\cdot)-2 g(\cdot+s)+g(\cdot+2 s)\|_{L_{2}(\mathbb{R})}
$$


(see 3 for properties and more detail). Using the above estimate with $g:=v^{n,-}$, we obtain

$$
\left\|\tilde{P}_{h} v^{n,-}-v^{n,-}\right\|_{L_{2}(\mathbb{R})} \leq C \omega_{2}\left(v^{n,-}, h\right)_{L_{2}(\mathbb{R})}=C \omega_{2}\left(v^{n-1}, h\right)_{L_{2}(\mathbb{R})}
$$

because $v^{n,-}=S_{a \Delta t} v^{n-1}$ and the modulus of smoothness $\omega_{2}\left(v^{n-1}, h\right)_{L_{2}(\mathbb{R})}$ is shift invariant. It is not easy to compute $\omega_{2}(g, h)_{L_{2}(\mathbb{R})}$ for a general $g$ but in the case of the piecewise linear function $v^{n}$ it is not hard to show that for any $n=0,1, \ldots, N$, we have

$$
C_{1} h^{1 / 2}\left\|\left\{\Delta^{2} v_{j}^{n}\right\}\right\|_{l_{2}} \leq \omega_{2}\left(v^{n}, h\right)_{L_{2}(\mathbb{R})} \leq C_{2} h^{1 / 2}\left\|\left\{\Delta^{2} v_{j}^{n}\right\}\right\|_{l_{2}},
$$

with absolute constants $C_{1}$ and $C_{2}$. Finally, we use (28) and (27) in (24) and obtain

$$
\left\|v^{n}-v^{n,-}\right\|_{L_{2}(\mathbb{R})} \leq C h^{1 / 2}\left(\left\|\left\{\Delta^{2} v_{j}^{n-1}\right\}\right\|_{l_{2}}+\left\|\left\{\Delta^{2} v_{j}^{n}\right\}\right\|_{l_{2}}\right) .
$$

We now go back and use (29) in the error estimate (22) to derive

$$
\left\|e^{N}\right\|_{-1,2} \leq C h^{3 / 2} \sum_{n=0}^{N}\left\|\left\{\Delta^{2} v_{j}^{n}\right\}\right\|_{l_{2}}=C h^{3 / 2} \sum_{n=0}^{N}\left\|\left\{\Delta \delta_{j}^{n}\right\}\right\|_{l_{2}} .
$$

Here we recall that $\delta_{j}^{n}=v_{j}^{n}-v_{j-1}^{n}$. Up to this point, our arguments are very similar to the ones in our previous paper [11]. The only difference is that here, in the estimate for the negative norm (30), we use $\left\|\left\{\Delta \delta_{j}^{n}\right\}\right\|_{l_{2}}$ instead of $\left\|\left\{\delta_{j}^{n}\right\}\right\|_{l_{2}}$ that was used in [11. A new idea here will be the use of the improved $l_{2}$-stability, Lemma 1, to derive a better error estimate than before. Let us recall the lower bound in the statement of Lemma 1]. For any $n=0, \ldots, N$, we have

$$
\sum_{j}\left(\delta_{j}^{n}\right)^{2}-\sum_{j}\left(\delta_{j}^{n+1}\right)^{2} \geq \frac{\beta^{3}}{4} \sum_{j}\left(\Delta^{2} \delta_{j}^{n}\right)^{2},
$$

where $\beta=\frac{1}{2} \alpha(1-\alpha)$. We sum (31) for $n=0, \ldots, N$ and obtain

$$
\frac{\beta^{3}}{4} \sum_{n=0}^{N} \sum_{j}\left(\Delta^{2} \delta_{j}^{n}\right)^{2} \leq \sum_{j}\left(\delta_{j}^{0}\right)^{2}-\sum_{j}\left(\delta_{j}^{N+1}\right)^{2} .
$$

Recall that the CFL condition is $0<\alpha<1$. In the trivial case $\alpha(1-\alpha)=0$, not covered by our theorem, we have a pure translation and the total error is equal to the error of the first step; i.e., the order of the error is $O(h)$. So, without loss of generality, we can assume that $0<\alpha<1$. Then, from (32), we derive

$$
\sum_{n=0}^{N} \sum_{j}\left(\Delta^{2} \delta_{j}^{n}\right)^{2} \leq C \sum_{j}\left(\delta_{j}^{0}\right)^{2},
$$

where $C$ is an absolute constant for any fixed $0<\alpha<1\left(C=4 \beta^{-3}\right)$. Using (33), we will now estimate $\sum_{n=0}^{N}\left\|\left\{\Delta \delta_{j}^{n}\right\}\right\|_{l_{2}}$. Because the support of $\frac{d u^{0}}{d x}$ is finite and the numerical solution has a finite domain of dependence, we have that the set $\Lambda_{n}:=\left\{j: \delta_{j}^{n} \neq 0\right\}$ is finite for all $n=0,1, \ldots, N$. Using Abel's transform and the finite support of $\left\{\delta_{j}^{n}\right\}$, we obtain

$$
\sum_{j}\left(\Delta \delta_{j}^{n}\right)^{2}=-\sum_{j} \delta_{j}^{n}\left(\delta_{j+1}^{n}-2 \delta_{j}^{n}+\delta_{j-1}^{n}\right) .
$$


Hence, we have

$$
\sum_{j}\left(\Delta \delta_{j}^{n}\right)^{2} \leq \sum_{j}\left|\delta_{j}^{n}\right|\left|\delta_{j+1}^{n}-2 \delta_{j}^{n}+\delta_{j-1}^{n}\right| \leq\left\|\left\{\delta_{j}^{n}\right\}\right\| l_{l_{2}}\left\|\left\{\Delta^{2} \delta_{j}^{n}\right\}\right\|_{l_{2}} .
$$

Using that $\left\|\left\{\delta_{j}^{n}\right\}\right\|_{l_{2}} \leq\left\|\left\{\delta_{j}^{0}\right\}\right\|_{l_{2}}$, we derive

$$
\left\|\left\{\Delta \delta_{j}^{n}\right\}\right\|_{l_{2}} \leq\left\|\left\{\delta_{j}^{0}\right\}\right\|_{l_{2}}^{1 / 2}\left\|\left\{\Delta^{2} \delta_{j}^{n}\right\}\right\|_{l_{2}}^{1 / 2} .
$$

Therefore, we have the estimates

$$
\begin{aligned}
\sum_{n=0}^{N}\left\|\left\{\Delta \delta_{j}^{n}\right\}\right\|_{l_{2}} & \leq\left\|\left\{\delta_{j}^{0}\right\}\right\|_{l_{2}}^{1 / 2} \sum_{n=0}^{N}\left\|\left\{\Delta^{2} \delta_{j}^{n}\right\}\right\|_{l_{2}}^{1 / 2} \\
& \leq\left\|\left\{\delta_{j}^{0}\right\}\right\|_{l_{2}}^{1 / 2}(N+1)^{3 / 4}\left(\sum_{n=0}^{N}\left\|\left\{\Delta^{2} \delta_{j}^{n}\right\}\right\|_{l_{2}}^{2}\right)^{1 / 4},
\end{aligned}
$$

where we use Hölder's inequality to derive (35). We use our stability estimate (33) in (35) and conclude

$$
\sum_{n=0}^{N}\left\|\left\{\Delta \delta_{j}^{n}\right\}\right\|_{l_{2}} \leq(N+1)^{3 / 4}\left\|\left\{\delta_{j}^{0}\right\}\right\|_{l_{2}} .
$$

From Lemma 4 in [11], we have that $\left\|\left\{\delta_{j}^{0}\right\}\right\|_{l_{2}} \leq h^{1 / 2}\left|u^{0}\right|_{W^{1}\left(L_{2}(\mathbb{R})\right)}$. Hence,

$$
\sum_{n=0}^{N}\left\|\left\{\Delta \delta_{j}^{n}\right\}\right\|_{l_{2}} \leq C N^{3 / 4} h^{1 / 2}\left|u^{0}\right|_{W^{1}\left(L_{2}(\mathbb{R})\right)} .
$$

We use (37) in (30) and derive the final minus-one norm error estimate

$$
\left\|e^{N}\right\|_{-1,2} \leq C(N h)^{3 / 4} h^{5 / 4}\left|u^{0}\right|_{W^{1}\left(L_{2}(\mathbb{R})\right)} \leq C h^{5 / 4}\left|u^{0}\right|_{W^{1}\left(L_{2}(\mathbb{R})\right)},
$$

where in the last inequality $C$ depends on $T$ and $\alpha$. Similar to [11, we will now interpolate between the negative norm estimate (38) and the discrete $W_{2}^{1}$-stability of the numerical scheme (11). Recall that $e^{N}=u(\cdot, T)-v^{N}$ is not in $W^{1}\left(L_{2}(\mathbb{R})\right)$. Analogous to [11, we approximate $v^{N}$ with $\tilde{v}$, the Steklov (sliding) average of the piecewise constant function $A_{h} v^{N}, A_{h} v^{N}(x)=v_{j}^{N}$ for $x \in\left[x_{j-1 / 2}, x_{j+1 / 2}\right), j \in \mathbb{Z}$. That is,

$$
\tilde{v}(x):=\frac{1}{h} \int_{x-h / 2}^{x+h / 2} A_{h} v^{N}(s) d s, \quad x \in \mathbb{R} .
$$

The function $\tilde{v} \in W_{\text {loc }}^{1}\left(L_{2}(\mathbb{R})\right)$ and has the properties

$$
\begin{aligned}
& \left\|\tilde{v}-v^{N}\right\|_{-1,2} \leq C h^{2}\left|u^{0}\right|_{W^{1}\left(L_{2}(\mathbb{R})\right)}, \\
& \left\|\tilde{v}-v^{N}\right\|_{L^{2}(\mathbb{R})} \leq C h\left|u^{0}\right|_{W^{1}\left(L_{2}(\mathbb{R})\right)},
\end{aligned}
$$

and

$$
|\tilde{v}|_{W^{1}\left(L_{2}(\mathbb{R})\right)} \leq\left|u^{0}\right|_{W^{1}\left(L_{2}(\mathbb{R})\right)}
$$

(see (54), (55), and (56) in [11]). Let $\tilde{e}:=u(\cdot, T)-\tilde{v}$. Then

$$
\|\tilde{e}\|_{-1,2} \leq\left\|e^{N}\right\|_{-1,2}+\left\|\tilde{v}-v^{N}\right\|_{-1,2} .
$$

Using (38) and (39), we obtain

$$
\|\tilde{e}\|_{-1,2} \leq C h^{5 / 4}\left|u^{0}\right|_{W^{1}\left(L_{2}(\mathbb{R})\right)} .
$$


Let $\tilde{E}(x)=\int_{-\infty}^{x} \tilde{e}(s) d s$. Then we have $\|\tilde{E}\|_{L_{2}}=\|\tilde{e}\|_{-1,2},\left\|\tilde{E}^{\prime}\right\|_{L_{2}}=\|\tilde{e}\|_{L_{2}}$, and

$$
\left\|\tilde{E}^{\prime \prime}\right\|_{L_{2}}=\left\|\tilde{e}^{\prime}\right\|_{L_{2}} \leq\left|u^{0}\right|_{W^{1}\left(L_{2}(\mathbb{R})\right)}+|\tilde{v}|_{W^{1}\left(L_{2}(\mathbb{R})\right)} \leq 2\left|u^{0}\right|_{W^{1}\left(L_{2}(\mathbb{R})\right)} .
$$

The Kolmogorov-Landau inequality in $L^{2}(\mathbb{R})$ (page 156 in 3 ) for the functions $\tilde{E}(x), \tilde{E}^{\prime}$, and $\tilde{E}^{\prime \prime}$ and the above estimate for $\left\|\tilde{E}^{\prime \prime}\right\|_{L_{2}}$ give

$$
\|\tilde{e}\|_{L_{2}}=\left\|\tilde{E}^{\prime}\right\|_{L_{2}} \leq \sqrt{2}\|\tilde{E}\|_{L_{2}}^{1 / 2}\left\|\tilde{E}^{\prime \prime}\right\|_{L_{2}}^{1 / 2} \leq 2\|\tilde{e}\|_{-1,2}^{1 / 2}\left|u^{0}\right|_{W^{1}\left(L_{2}(\mathbb{R})\right)}^{1 / 2} .
$$

Using (42), we derive

$$
\|\tilde{e}\|_{L_{2}} \leq C h^{5 / 8}\left|u^{0}\right|_{W^{1}\left(L_{2}(\mathbb{R})\right)} .
$$

By the triangle inequality, we have

$$
\left\|e^{N}\right\|_{L_{2}} \leq\|\tilde{e}\|_{L_{2}}+\left\|v^{N}-\tilde{v}\right\|_{L_{2}},
$$

and using the estimates (43) and (40) we conclude

$$
\left\|e^{N}\right\|_{L_{2}} \leq C h^{5 / 8}\left|u^{0}\right|_{W^{1}\left(L_{2}(\mathbb{R})\right)}
$$

where the constant $C$ depends only on $T$ and $\alpha$.

\section{Appendix: Proof of Lemma 1}

Let us recall the statement of the lemma. We claim (see (12)) that

$$
3 \beta \sum_{j}\left(\Delta^{2} \delta_{j}\right)^{2} \geq \sum_{j} \delta_{j}^{2}-\sum_{j}\left(\delta_{j}^{\prime}\right)^{2} \geq \frac{\beta^{3}}{4} \sum_{j}\left(\Delta^{2} \delta_{j}\right)^{2}
$$

where $\beta=\frac{1}{2} \alpha(1-\alpha)$.

It is enough to consider the case of a nondecreasing data, $\delta_{j} \geq 0$, with the other case being analogous. Let us introduce some notation. Let $y_{j}=\min \left(\delta_{j}, \delta_{j+1}\right)$, $\Delta \delta_{j}=\delta_{j}-\delta_{j-1}, \Delta y_{j}=y_{j}-y_{j-1}, \Delta^{2} \delta_{j}=\delta_{j}-2 \delta_{j-1}+\delta_{j-2}$, and $\Delta^{2} y_{j}=y_{j}-$ $2 y_{j-1}+y_{j-2}$. Recall that $\left\{\delta_{j}\right\}_{-\infty}^{\infty} \in l_{2}$, and $\delta_{j} \geq 0$ for all $j$. It is enough to prove Lemma 1 only for $0<\alpha<1$. We construct the new sequence $\left\{\delta_{j}^{\prime}\right\}$ by using the rule

$$
\delta_{j}^{\prime}=(1-\alpha) \delta_{j}+\alpha \delta_{j-1}-\beta \Delta^{2} y_{j},
$$

for each $j$. First we assume that $\left\{\delta_{j}\right\}$ has finite support. It is easy to see how to modify the proof in case the support is not finite. Therefore we assume $\delta_{j}=0$ for $j \leq 3$ and for $j \geq M-3$ for some integer $M$. Then $\delta_{j}^{\prime}=0$ for $j \leq 3$ and $j \geq M-2$. Thus

$$
\left\|\left\{\delta_{j}\right\}\right\|_{l_{2}}^{2}-\left\|\left\{\delta_{j}^{\prime}\right\}\right\|_{l_{2}}^{2}=\sum_{j=1}^{M} \delta_{j}^{2}-\sum_{j=1}^{M}\left(\delta_{j}^{\prime}\right)^{2}
$$

We have

$$
\sum_{j=1}^{M}\left(\delta_{j}^{\prime}\right)^{2}=\sum_{j=1}^{M}\left(\left((1-\alpha) \delta_{j}+\alpha \delta_{j-1}\right)^{2}-2 \beta\left((1-\alpha) \delta_{j}+\alpha \delta_{j-1}\right) \Delta^{2} y_{j}+\beta^{2}\left(\Delta^{2} y_{j}\right)^{2}\right) .
$$


Note that since $\delta_{0}=\delta_{1}=0$ and $\delta_{M-1}=\delta_{M}=0$, we have

$$
\begin{aligned}
\sum_{j=1}^{M} \delta_{j}^{2}-\left((1-\alpha) \delta_{j}+\alpha \delta_{j-1}\right)^{2} & \\
= & \sum_{j=1}^{M}\left(\left(1-(1-\alpha)^{2}\right) \delta_{j}^{2}-2 \alpha(1-\alpha) \delta_{j} \delta_{j-1}-\alpha^{2} \sum_{j=1}^{M} \delta_{j-1}^{2}\right. \\
= & \sum_{j=1}^{M}\left(1-(1-\alpha)^{2}-\alpha^{2}\right) \delta_{j}^{2}-2 \alpha(1-\alpha) \delta_{j} \delta_{j-1} \\
= & 2 \beta \sum_{j=1}^{M}\left(2 \delta_{j}^{2}-2 \delta_{j} \delta_{j-1}\right)=2 \beta \sum_{j=1}^{M}\left(\delta_{j}-\delta_{j-1}\right)^{2}=2 \beta \sum_{j=1}^{M}\left(\Delta \delta_{j}\right)^{2} .
\end{aligned}
$$

Therefore

$$
\sum_{j=1}^{M} \delta_{j}^{2}-\left((1-\alpha) \delta_{j}+\alpha \delta_{j-1}\right)^{2}=2 \beta \sum_{j=1}^{M}\left(\Delta \delta_{j}\right)^{2}
$$

Thus we get

$$
\sum_{j=1}^{M} \delta_{j}^{2}-\sum_{j=1}^{M}\left(\delta_{j}^{\prime}\right)^{2}=2 \beta \sum_{j=1}^{M}\left(\left(\Delta \delta_{j}\right)^{2}+\left((1-\alpha) \delta_{j}+\alpha \delta_{j-1}\right) \Delta^{2} y_{j}-\frac{\beta}{2}\left(\Delta^{2} y_{j}\right)^{2}\right) .
$$

Now we use $\Delta^{2} y_{j}=\Delta y_{j}-\Delta y_{j-1}, \Delta y_{j}=0, \delta_{j}=0$ for $j \leq 1, j \geq M-1$, and Abel's transform to obtain

$$
\sum_{j=1}^{M} \delta_{j} \Delta^{2} y_{j}=\sum_{j=1}^{M}\left(\delta_{j}-\delta_{j+1}\right) \Delta y_{j}
$$

and

$$
\sum_{j=1}^{M} \delta_{j-1} \Delta^{2} y_{j}=\sum_{j=1}^{M}\left(\delta_{j-1}-\delta_{j}\right) \Delta y_{j}
$$

So, (47) becomes

$$
\begin{gathered}
\sum_{j=1}^{M} \delta_{j}^{2}-\sum_{j=1}^{M}\left(\delta_{j}^{\prime}\right)^{2}=2 \beta\left(\sum_{j=1}^{M}\left(\Delta \delta_{j}\right)^{2}-(1-\alpha) \sum_{j=1}^{M} \Delta \delta_{j+1} \Delta y_{j}\right. \\
\left.\quad-\alpha \sum_{j=1}^{M} \Delta \delta_{j} \Delta y_{j}-\frac{\beta}{2} \sum_{j=1}^{M}\left(\Delta^{2} y_{j}\right)^{2}\right) \\
=2 \beta Q_{1}+\beta^{2} Q_{2},
\end{gathered}
$$

where

$$
Q_{1}=\sum_{j=1}^{M}\left(\Delta \delta_{j}\right)^{2}-(1-\alpha) \sum_{j=1}^{M} \Delta \delta_{j+1} \Delta y_{j}-\alpha \sum_{j=1}^{M} \Delta \delta_{j} \Delta y_{j}-\beta \sum_{j=1}^{M}\left(\Delta^{2} \delta_{j}\right)^{2},
$$

and

$$
Q_{2}=2 \sum_{j=1}^{M}\left(\Delta^{2} \delta_{j}\right)^{2}-\sum_{j=1}^{M}\left(\Delta^{2} y_{j}\right)^{2}
$$


To finish the proof, it is sufficient prove the following three lemmas.

\section{Lemma 2.}

$$
\begin{aligned}
Q_{1}= & \left.\frac{1}{2} \sum_{\Delta \delta_{j}<0, \Delta \delta_{j-1} \geq 0}\left((1-\alpha) \Delta \delta_{j-1}+\alpha \Delta \delta_{j}\right)\right)^{2} \\
& \left.+\frac{1}{2} \sum_{\Delta \delta_{j} \geq 0, \Delta \delta_{j-1}<0}\left((1-\alpha) \Delta \delta_{j}+\alpha \Delta \delta_{j-1}\right)\right)^{2} \\
& -\sum_{\Delta \delta_{j} \geq 0, \Delta \delta_{j+1}<0} \Delta \delta_{j} \Delta \delta_{j+1} \\
& +\left(\frac{1-\alpha}{2}-\beta\right) \sum_{\Delta \delta_{j-1} \geq 0, \Delta \delta_{j} \geq 0}\left(\Delta^{2} \delta_{j}\right)^{2} \\
& +\left(\frac{\alpha}{2}-\beta\right) \sum_{\Delta \delta_{j-1}<0, \Delta \delta_{j}<0}\left(\Delta^{2} \delta_{j}\right)^{2} \geq 0 .
\end{aligned}
$$

\section{Lemma 3.}

$$
\begin{aligned}
Q_{2}= & \left.\sum_{j}\left(\left(\Delta \delta_{j+1}\right)_{-}-\Delta \delta_{j}+\left(\Delta \delta_{j-1}\right)_{+}\right)\right)^{2} \\
& -2 \sum_{j} \Delta \delta_{j} \Delta \delta_{j-1}\left(1-\operatorname{sgn}\left(\Delta \delta_{j} \Delta \delta_{j-1}\right)\right) \geq 0 .
\end{aligned}
$$

Here $\operatorname{sgn}(x)$ is the usual sign function defined by

$$
\operatorname{sgn}(x)=\left\{\begin{array}{cl}
1, & \text { if } \quad x>0 \\
0, & \text { if } x=0 \\
-1, & \text { if } x<0
\end{array}\right.
$$

and $u_{+}=\max (u, 0), u_{-}=\min (u, 0)$.

\section{Lemma 4.}

$$
3 \beta \sum_{j=1}^{M}\left(\Delta \delta_{j}\right)^{2} \geq 2 \beta Q_{1}+\beta^{2} Q_{2} \geq \frac{\beta^{3}}{4} \sum_{j=1}^{M}\left(\Delta \delta_{j}\right)^{2} .
$$

In order to simplify our notation, we will use $\sum_{j}$ instead of $\sum_{j=1}^{M}$ in the proofs of all lemmas.

\section{Proof of Lemma 2. Denote}

$$
A=\sum_{j} \Delta \delta_{j+1} \Delta y_{j} \quad \text { and } \quad B=\sum_{j} \Delta \delta_{j} \Delta y_{j} .
$$

Our aim is to rewrite

$$
Q_{1}=\sum_{j}\left(\Delta \delta_{j}\right)^{2}-(1-\alpha) A-\alpha B-\beta \sum_{j}\left(\Delta^{2} \delta_{j}\right)^{2}
$$

in the form indicated in Lemma 2. It is easy to check that

$$
\Delta y_{j}=\left(\Delta \delta_{j}\right)_{+}+\left(\Delta \delta_{j+1}\right)_{-} .
$$


We can transform $A$ as follows:

$$
\begin{aligned}
A & =\sum_{j} \Delta \delta_{j+1}\left(\left(\Delta \delta_{j}\right)_{+}+\left(\Delta \delta_{j+1}\right)_{-}\right) \\
& =\sum_{j} \Delta \delta_{j+1}\left(\Delta \delta_{j}\right)_{+}+\sum_{j} \Delta \delta_{j}\left(\Delta \delta_{j}\right)_{-} \\
& =\sum_{\Delta \delta_{j}<0}\left(\Delta \delta_{j}\right)^{2}+\sum_{\Delta \delta_{j} \geq 0} \Delta \delta_{j} \Delta \delta_{j+1} \\
& =\sum_{\Delta \delta_{j}<0}\left(\Delta \delta_{j}\right)^{2}+\sum_{\Delta \delta_{j} \geq 0, \Delta \delta_{j+1}<0} \Delta \delta_{j} \Delta \delta_{j+1}+D,
\end{aligned}
$$

where

$$
D=\sum_{\Delta \delta_{j} \geq 0, \Delta \delta_{j+1} \geq 0} \Delta \delta_{j} \Delta \delta_{j+1}
$$

Further,

$$
\begin{aligned}
D & =\frac{1}{2} \sum_{\Delta \delta_{j} \geq 0, \Delta \delta_{j+1} \geq 0}\left(\left(\Delta \delta_{j}\right)^{2}+\left(\Delta \delta_{j+1}\right)^{2}-\left(\Delta^{2} \delta_{j+1}\right)^{2}\right) \\
& =\frac{1}{2} \sum_{\Delta \delta_{j-1} \geq 0, \Delta \delta_{j} \geq 0}\left(\left(\Delta \delta_{j-1}\right)^{2}+\left(\Delta \delta_{j}\right)^{2}-\left(\Delta^{2} \delta_{j}\right)^{2}\right) \\
& =\frac{1}{2} \sum_{\Delta \delta_{j} \geq 0, \Delta \delta_{j+1} \geq 0}\left(\Delta \delta_{j}\right)^{2}+\frac{1}{2} \sum_{\Delta \delta_{j} \geq 0, \Delta \delta_{j-1} \geq 0}\left(\Delta \delta_{j}\right)^{2}-\frac{1}{2} \sum_{\Delta \delta_{j-1} \geq 0, \Delta \delta_{j} \geq 0}\left(\Delta^{2} \delta_{j}\right)^{2} .
\end{aligned}
$$

Also, note that

$$
\sum_{\Delta \delta_{j} \geq 0, \Delta \delta_{j+1} \geq 0}\left(\Delta \delta_{j}\right)^{2}=\sum_{\Delta \delta_{j} \geq 0}\left(\Delta \delta_{j}\right)^{2}-\sum_{\Delta \delta_{j} \geq 0, \Delta \delta_{j+1}<0}\left(\Delta \delta_{j}\right)^{2}
$$

and

$$
\sum_{\Delta \delta_{j} \geq 0, \Delta \delta_{j-1} \geq 0}\left(\Delta \delta_{j}\right)^{2}=\sum_{\Delta \delta_{j} \geq 0}\left(\Delta \delta_{j}\right)^{2}-\sum_{\Delta \delta_{j} \geq 0, \Delta \delta_{j-1}<0}\left(\Delta \delta_{j}\right)^{2} .
$$

By (500), (51), and (52) we get

$$
\begin{aligned}
A= & \sum_{j}\left(\Delta \delta_{j}\right)^{2}-\frac{1}{2} \sum_{\Delta \delta_{j} \geq 0, \Delta \delta_{j+1}<0}\left(\Delta \delta_{j}\right)^{2}-\frac{1}{2} \sum_{\Delta \delta_{j} \geq 0, \Delta \delta_{j-1}<0}\left(\Delta \delta_{j}\right)^{2} \\
& -\frac{1}{2} \sum_{\Delta \delta_{j-1} \geq 0, \Delta \delta_{j} \geq 0}\left(\Delta^{2} \delta_{j}\right)^{2}+\sum_{\Delta \delta_{j} \geq 0, \Delta \delta_{j+1}<0} \Delta \delta_{j} \Delta \delta_{j+1} .
\end{aligned}
$$

Transform $B$ in the same way as $A$ :

$$
B=\sum_{\Delta \delta_{j} \geq 0}\left(\Delta \delta_{j}\right)^{2}+\sum_{\Delta \delta_{j} \geq 0, \Delta \delta_{j+1}<0} \Delta \delta_{j} \Delta \delta_{j+1}+E,
$$

where

$$
E=\sum_{\Delta \delta_{j}<0, \Delta \delta_{j+1}<0} \Delta \delta_{j} \Delta \delta_{j+1} .
$$

The quantity $E$ can also be rewritten in the same way as $D$ :

$$
E=\frac{1}{2} \sum_{\Delta \delta_{j}<0, \Delta \delta_{j+1}<0}\left(\Delta \delta_{j}\right)^{2}+\frac{1}{2} \sum_{\Delta \delta_{j-1}<0, \Delta \delta_{j}<0}\left(\Delta \delta_{j}\right)^{2}-\frac{1}{2} \sum_{\Delta \delta_{j-1}<0, \Delta \delta_{j}<0}\left(\Delta^{2} \delta_{j}\right)^{2} .
$$


Combining this equality with (54) we get

$$
\begin{aligned}
B= & \sum_{j}\left(\Delta \delta_{j}\right)^{2}-\frac{1}{2} \sum_{\Delta \delta_{j}<0, \Delta \delta_{j+1} \geq 0}\left(\Delta \delta_{j}\right)^{2}-\frac{1}{2} \sum_{\Delta \delta_{j}<0, \Delta \delta_{j-1} \geq 0}\left(\Delta \delta_{j}\right)^{2} \\
& -\frac{1}{2} \sum_{\Delta \delta_{j-1}<0, \Delta \delta_{j}<0}\left(\Delta^{2} \delta_{j}\right)^{2}+\sum_{\Delta \delta_{j} \geq 0, \Delta \delta_{j+1}<0} \Delta \delta_{j} \Delta \delta_{j+1} .
\end{aligned}
$$

By (53) and (55),

$$
Q_{1}=\sum_{j}\left(\Delta \delta_{j}\right)^{2}-(1-\alpha) A-\alpha B-\beta \sum_{j}\left(\Delta^{2} \delta_{j}\right)^{2}=\sum_{i=1}^{9} S_{i},
$$

where

$$
\begin{gathered}
S_{1}=\frac{1-\alpha}{2} \sum_{\Delta \delta_{j} \geq 0, \Delta \delta_{j+1}<0}\left(\Delta \delta_{j}\right)^{2}, \quad S_{2}=\frac{1-\alpha}{2} \sum_{\Delta \delta_{j} \geq 0, \Delta \delta_{j-1}<0}\left(\Delta \delta_{j}\right)^{2}, \\
S_{3}=\frac{\alpha}{2} \sum_{\Delta \delta_{j}<0, \Delta \delta_{j+1} \geq 0}\left(\Delta \delta_{j}\right)^{2}, \quad S_{4}=\frac{\alpha}{2} \sum_{\Delta \delta_{j}<0, \Delta \delta_{j-1} \geq 0}\left(\Delta \delta_{j}\right)^{2}, \\
S_{5}=-\sum_{\Delta \delta_{j} \geq 0, \Delta \delta_{j+1}<0} \Delta \delta_{j} \Delta \delta_{j+1}, \\
S_{6}=\left(\frac{1-\alpha}{2}-\beta\right) \sum_{\Delta \delta_{j-1} \geq 0, \Delta \delta_{j} \geq 0}\left(\Delta^{2} \delta_{j}\right)^{2}, \quad S_{7}=\left(\frac{\alpha}{2}-\beta\right) \sum_{\Delta \delta_{j-1}<0, \Delta \delta_{j}<0}\left(\Delta^{2} \delta_{j}\right)^{2}, \\
S_{8}=-\beta \quad \sum_{\Delta \delta_{j-1} \geq 0, \Delta \delta_{j}<0}\left(\Delta^{2} \delta_{j}\right)^{2}, \quad \text { and } \quad S_{9}=-\beta \sum_{\Delta \delta_{j-1}<0, \Delta \delta_{j} \geq 0}\left(\Delta^{2} \delta_{j}\right)^{2} .
\end{gathered}
$$

To get an upper bound for $Q_{1}$, note that $\left|S_{j}\right| \leq \frac{1}{2} \sum_{j}\left(\Delta^{2} \delta_{j}\right)^{2}$ for $j=1,2,3,4,5$. Therefore, $Q_{1} \leq(3-2 \beta) \sum_{j}\left(\Delta^{2} \delta_{j}\right)^{2}$. Also, from the definition of $Q_{2}, Q_{2} \leq$ $2 \sum_{j}\left(\Delta^{2} \delta_{j}\right)^{2}$. Combining the upper bounds for $Q_{1}$ and $Q_{2}$ we get the upper bound in Lemma 1 .

Next, note that

$$
S_{1}+S_{4}+S_{8}=\sum_{\Delta \delta_{j}<0, \Delta \delta_{j-1} \geq 0}\left(\frac{1-\alpha}{2}\left(\Delta \delta_{j-1}\right)^{2}+\frac{\alpha}{2}\left(\Delta \delta_{j}\right)^{2}-\beta\left(\Delta^{2} \delta_{j}\right)^{2}\right) .
$$

Also,

$$
\begin{aligned}
& \frac{1-\alpha}{2}\left(\Delta \delta_{j-1}\right)^{2}+\frac{\alpha}{2}\left(\Delta \delta_{j}\right)^{2}-\beta\left(\Delta^{2} \delta_{j}\right)^{2} \\
& \quad=\frac{1-\alpha}{2}\left(\Delta \delta_{j-1}\right)^{2}+\frac{\alpha}{2}\left(\Delta \delta_{j}\right)^{2}-\frac{1}{2}(1-\alpha) \alpha\left(\Delta \delta_{j}-\Delta \delta_{j-1}\right)^{2} \\
& \left.\quad=\frac{1}{2}\left((1-\alpha) \Delta \delta_{j-1}+\alpha \Delta \delta_{j}\right)\right)^{2} .
\end{aligned}
$$

Summing the last inequality over all $j$ with $\Delta \delta_{j-1} \geq 0, \Delta \delta_{j}<0$, we get

$$
\left.S_{1}+S_{4}+S_{8}=\frac{1}{2} \sum_{\Delta \delta_{j}<0, \Delta \delta_{j-1} \geq 0}\left((1-\alpha) \Delta \delta_{j-1}+\alpha \Delta \delta_{j}\right)\right)^{2} .
$$

Similarly,

$$
S_{2}+S_{3}+S_{9}=\sum_{\Delta \delta_{j} \geq 0, \Delta \delta_{j-1}<0}\left(\frac{1-\alpha}{2}\left(\Delta \delta_{j}\right)^{2}+\frac{\alpha}{2}\left(\Delta \delta_{j-1}\right)^{2}-\beta\left(\Delta^{2} \delta_{j}\right)^{2}\right) .
$$


As before,

$$
\frac{1-\alpha}{2}\left(\Delta \delta_{j}\right)^{2}+\frac{\alpha}{2}\left(\Delta \delta_{j-1}\right)^{2}-\beta\left(\Delta^{2} \delta_{j}\right)^{2}=\frac{1}{2}\left(\alpha \Delta \delta_{j-1}+(1-\alpha) \Delta \delta_{j}\right)^{2} .
$$

Summing the last inequality over all $j$ with $\Delta \delta_{j} \geq 0, \Delta \delta_{j-1}<0$, we get

$$
S_{2}+S_{3}+S_{9}=\frac{1}{2} \sum_{\Delta \delta_{j} \geq 0, \Delta \delta_{j-1}<0}\left(\alpha \Delta \delta_{j-1}+(1-\alpha) \Delta \delta_{j}\right)^{2} .
$$

This completes the proof of Lemma 2 .

Proof of Lemma 3, By (49) we have $\Delta y_{j}=\left(\Delta \delta_{j}\right)_{+}+\left(\Delta \delta_{j+1}\right)_{-}$. We also have $\Delta^{2} \delta_{j}=\Delta \delta_{j}-\Delta \delta_{j-1}$. Thus, we need to rewrite the quantity

$$
Q_{2}=2 \sum_{j}\left(\Delta \delta_{j}-\Delta \delta_{j-1}\right)^{2}-\sum_{j}\left(\left(\left(\Delta \delta_{j+1}\right)_{-}-\left(\Delta \delta_{j}\right)_{-}\right)+\left(\left(\Delta \delta_{j}\right)_{+}-\left(\Delta \delta_{j-1}\right)_{+}\right)\right)^{2}
$$

in the form indicated in Lemma 3. We now use that for any real numbers $x$ and $y$, $(x+y)^{2}=2 x^{2}+2 y^{2}-(x-y)^{2}$, and get

$$
\begin{aligned}
\sum_{j}(( & \left.\left.\left(\Delta \delta_{j+1}\right)_{-}-\left(\Delta \delta_{j}\right)_{-}\right)+\left(\left(\Delta \delta_{j}\right)_{+}-\left(\Delta \delta_{j-1}\right)_{+}\right)\right)^{2} \\
= & 2\left(\sum_{j}\left(\left(\Delta \delta_{j+1}\right)_{-}-\left(\Delta \delta_{j}\right)_{-}\right)^{2}+\sum_{j}\left(\left(\Delta \delta_{j}\right)_{+}-\left(\Delta \delta_{j-1}\right)_{+}\right)^{2}\right) \\
& -\sum_{j}\left(\left(\Delta \delta_{j+1}\right)_{-}-\left(\Delta \delta_{j}\right)_{-}-\left(\Delta \delta_{j}\right)_{+}+\left(\Delta \delta_{j-1}\right)_{+}\right)^{2} \\
= & 2\left(\sum_{j}\left(\left(\Delta \delta_{j}\right)_{-}-\left(\Delta \delta_{j-1}\right)_{-}\right)^{2}+\sum_{j}\left(\left(\Delta \delta_{j}\right)_{+}-\left(\Delta \delta_{j-1}\right)_{+}\right)^{2}\right) \\
& -\sum_{j}\left(\left(\Delta \delta_{j+1}\right)_{-}-\Delta \delta_{j}+\left(\Delta \delta_{j-1}\right)_{+}\right)^{2}
\end{aligned}
$$

(in the last equality we have used that the sequence $\left\{\Delta \delta_{j}\right\}$ has finite support).

Now, we claim that for any real numbers $x$ and $y$

$$
(x-y)^{2}+x y(1-\operatorname{sgn}(x y))=\left(x_{-}-y_{-}\right)^{2}+\left(x_{+}-y_{+}\right)^{2} .
$$

First, if $x=0$, both sides of (60) equal $y^{2}$. Similarly, if $y=0$, both sides of (60) equal $x^{2}$. Second, if $x$ and $y$ have opposite signs $(\operatorname{sgn}(x y)=-1)$, the LHS of (60) is $(x-y)^{2}+2 x y$ and the RHS is $x^{2}+y^{2}$. Finally, if $x$ and $y$ have the same sign then both the LHS and the RHS of (60) equal $(x-y)^{2}$. Therefore, we have shown that (60) holds in all cases.

By using (60) with $x=\Delta \delta_{j}, y=\Delta \delta_{j-1}$, and summing over $j$ we get

$$
\begin{aligned}
\sum_{j}\left(\left(\Delta \delta_{j}\right)_{-}-\left(\Delta \delta_{j-1}\right)_{-}\right)^{2} & +\sum_{j}\left(\left(\Delta \delta_{j}\right)_{+}-\left(\Delta \delta_{j-1}\right)_{+}\right)^{2} \\
=\sum_{j}\left(\Delta \delta_{j}-\Delta \delta_{j-1}\right)^{2} & +\sum_{j} \Delta \delta_{j} \Delta \delta_{j-1}\left(1-\operatorname{sgn}\left(\Delta \delta_{j} \Delta \delta_{j-1}\right)\right) .
\end{aligned}
$$

Combining (59) and (61) completes the proof of Lemma 3. 
Proof of Lemma 4. We proved the upper bound of Lemma 4 earlier, so we concentrate on the lower bound.

Denote the five sums appearing in Lemma 2 by $\Sigma_{1}, \ldots, \Sigma_{5}$, and the two sums appearing in Lemma 3 by $\Sigma_{6}$, and $\Sigma_{7}$. Also, let

$$
\mathcal{A}_{++}=\left\{j: \Delta \delta_{j} \geq 0, \Delta \delta_{j-1} \geq 0\right\}, \quad \mathcal{A}_{+-}=\left\{j: \Delta \delta_{j} \geq 0, \Delta \delta_{j-1}<0\right\},
$$

and

$$
\mathcal{A}_{-+}=\left\{j: \Delta \delta_{j}<0, \Delta \delta_{j-1} \geq 0\right\}, \quad \mathcal{A}_{--}=\left\{j: \Delta \delta_{j}<0, \Delta \delta_{j-1}<0\right\} .
$$

Since $\frac{1-\alpha}{2}-\beta=\frac{(1-\alpha)^{2}}{2}>\frac{\beta^{2}}{2}$ and $\frac{\alpha}{2}-\beta=\frac{\alpha^{2}}{2}>\frac{\beta^{2}}{2}$, we get

$$
\Sigma_{4}>\frac{\beta^{2}}{2} \sum_{j \in \mathcal{A}_{++}}\left(\Delta^{2} \delta_{j}\right)^{2} \quad \text { and } \quad \Sigma_{5}>\frac{\beta^{2}}{2} \sum_{j \in \mathcal{A}_{--}}\left(\Delta^{2} \delta_{j}\right)^{2} .
$$

Next, we need to divide both $\mathcal{A}_{+-}$and $\mathcal{A}_{-+}$to a "good" part and a "bad" part. We define the following index sets:

$$
\begin{aligned}
& \mathcal{A}_{+-}^{g}=\left\{j \in \mathcal{A}_{+-}:\left|(1-\alpha) \Delta \delta_{j-1}+\alpha \Delta \delta_{j}\right| \geq \frac{1}{2} \max \left((1-\alpha)\left|\Delta \delta_{j-1}\right|, \alpha\left|\Delta \delta_{j}\right|\right)\right\}, \\
& \mathcal{A}_{+-}^{b}=\left\{j \in \mathcal{A}_{+-}:\left|(1-\alpha) \Delta \delta_{j-1}+\alpha \Delta \delta_{j}\right|<\frac{1}{2} \max \left((1-\alpha)\left|\Delta \delta_{j-1}\right|, \alpha\left|\Delta \delta_{j}\right|\right)\right\}, \\
& \mathcal{A}_{-+}^{g}=\left\{j \in \mathcal{A}_{-+}:\left|(1-\alpha) \Delta \delta_{j}+\alpha \Delta \delta_{j-1}\right| \geq \frac{1}{2} \max \left((1-\alpha)\left|\Delta \delta_{j}\right|, \alpha\left|\Delta \delta_{j-1}\right|\right)\right\}, \\
& \mathcal{A}_{-+}^{b}=\left\{j \in \mathcal{A}_{-+}:\left|(1-\alpha) \Delta \delta_{j}+\alpha \Delta \delta_{j-1}\right|<\frac{1}{2} \max \left((1-\alpha)\left|\Delta \delta_{j}\right|, \alpha\left|\Delta \delta_{j-1}\right|\right)\right\} .
\end{aligned}
$$

Now, when $j \in \mathcal{A}_{+-}^{g}$ we have

$$
\begin{aligned}
\left|(1-\alpha) \Delta \delta_{j-1}+\alpha \Delta \delta_{j}\right| & \geq \frac{1}{2} \max \left((1-\alpha)\left|\Delta \delta_{j-1}\right|, \alpha\left|\Delta \delta_{j}\right|\right) \\
& >\beta \max \left(\left|\Delta \delta_{j-1}\right|,\left|\Delta \delta_{j}\right|\right) \geq \frac{\beta}{2}\left|\Delta^{2} \delta_{j}\right| .
\end{aligned}
$$

Therefore, $\Sigma_{2}>\frac{\beta^{2}}{8} \sum_{j \in \mathcal{A}_{+-}^{g}}\left(\Delta^{2} \delta_{j}\right)^{2}$. Exactly in the same way, we get $\Sigma_{1}>$ $\frac{\beta^{2}}{8} \sum_{j \in \mathcal{A}_{-+}^{g}}\left(\Delta^{2} \delta_{j}\right)^{2}$.

Next use that

$$
\text { If }|x+y|<\frac{1}{2} \max (|x|,|y|), \text { then } \frac{1}{2}<\left|\frac{x}{y}\right|<2 .
$$

Indeed, by symmetry it is sufficient to consider the case $|y| \geq|x|$. In this case $|y|-|x| \leq|x+y|<\frac{1}{2} \max (|x|,|y|)=\frac{1}{2}|y|$, and we get $\frac{1}{2}|y|<|x|$, so $\frac{1}{2}<\left|\frac{x}{y}\right|$, completing the proof of (62).

Next, let $j \in \mathcal{A}_{+-}^{b}$. Using (662) we obtain $\frac{1}{2}<\frac{(1-\alpha)\left|\Delta \delta_{j-1}\right|}{\alpha\left|\Delta \delta_{j}\right|}<2$. So,

$$
\frac{\left(\left|\Delta \delta_{j}\right|+\left|\Delta \delta_{j-1}\right|\right)^{2}}{\left|\Delta \delta_{j}\right|\left|\Delta \delta_{j-1}\right|}=\frac{\left|\Delta \delta_{j}\right|}{\left|\Delta \delta_{j-1}\right|}+2+\frac{\left|\Delta \delta_{j-1}\right|}{\left|\Delta \delta_{j}\right|}<\frac{2(1-\alpha)}{\alpha}+2+\frac{2 \alpha}{1-\alpha}=\frac{1-2 \beta}{\beta} .
$$


We get

$$
\left(\Delta^{2} \delta_{j}\right)^{2} \leq\left(\left|\Delta \delta_{j}\right|+\left|\Delta \delta_{j-1}\right|\right)^{2}<\frac{1-2 \beta}{\beta}\left|\Delta \delta_{j}\right|\left|\Delta \delta_{j-1}\right| .
$$

Exactly in the same way, one can show that (63) holds when $j \in \mathcal{A}_{-+}^{b}$. Also, note that $\mathcal{A}_{+-}^{b} \cap \mathcal{A}_{-+}^{b}=\emptyset$ and if $j \in \mathcal{A}_{+-}^{b} \cup \mathcal{A}_{-+}^{b}$, then $\operatorname{sgn}\left(\Delta \delta_{j} \Delta \delta_{j-1}\right)=-1$, and we get

$$
\Sigma_{7}>4 \beta\left(\sum_{j \in \mathcal{A}_{+-}^{b}}\left(\Delta^{2} \delta_{j}\right)^{2}+\sum_{j \in \mathcal{A}_{-+}^{b}}\left(\Delta^{2} \delta_{j}\right)^{2}\right) .
$$

Combining the lower bounds for $\Sigma_{4}, \Sigma_{5}, \Sigma_{2}, \Sigma_{1}$, and $\Sigma_{7}$, we get the lower bound in Lemma 4 (note that all $\Sigma$ 's are nonnegative). This completes the proof of Lemma 4 and of Lemma 1 .

\section{ACKNOWLEDGMENTS}

The authors are grateful to Ronald DeVore for his inspiring discussions and constant support. The authors also thank the anonymous referees. Their comments and suggestions helped improve the paper.

\section{REFERENCES}

[1] Y. Brenier and S. Osher, The one-sided Lipschitz condition for convex scalar conservation laws, SIAM J. Numer. Anal., 25: 8-23, 1988. MR0923922 (89a:65134)

[2] P. Brenner, V. Thomée and L. B. Wahlbin, Besov spaces and applications to difference methods for initial value problems, (A. Dold and B. Eckmann, eds.) Lecture Notes in Math., vol. 434, Springer-Verlag, Berlin and New York, 1975. MR0461121 (57:1106)

[3] R. A. DeVore and G. G. Lorentz, Constructive Approximation, Springer-Verlag, Berlin, 1993. MR1261635 (95f:41001)

[4] J. Goodman and R. LeVeque, A geometric approach to high resolution TVD schemes, SIAM J. Numer. Anal., 25: 268-284, 1988. MR0933724 (89c:65097)

[5] A. Harten and S. Osher, Uniformly high order accurate non-oscillatory schemes, I, SIAM J. Numer. Anal. 24: 279-309, 1987. MR0881365 (90a:65198)

[6] A. Harten, B. Enquist, S. Osher and S.R. Chakravarthy, Uniformly high order accurate essentially non-oscillatory schemes, III, J. Comp. Phys., 71: 231-303, 1987. MR0897244 (90a:65199)

[7] G.-S. Jiang, D. Levi, C.-T. Lin, S. Osher and E. Tadmor, High-resolution non-oscillatory central schemes with nonstaggered grids for hyperbolic conservation laws, SIAM J. Numer. Anal., 35: 2147-2169, 1998. MR1655841(99j:65145)

[8] G.-S. Jiang and E. Tadmor, Nonoscillatory central schemes for hyperbolic conservation laws, SIAM J. Sci. Comput., 19: 1892-1917, 1998. MR1638064 (99f:65128)

[9] Yu. V. Kryakin, On the theorem of H. Whitney in spaces $L^{p}, 1 \leq p \leq \infty$, Mathematica Balkanika, New series, Vol. 4, Fasc. 3: 258-270, 1990. MR1169221 (93i:41002)

[10] P. Lax and B. Wendroff, Systems of conservation laws, Comm. Pure Appl. Math., 13: 217237, 1960. MR0120774 (22:11523)

[11] S. Konyagin, B. Popov and O. Trifonov, On Convergence of Minmod-Type Schemes, SIAM J. Numer. Anal., 42: 1978-1997, 2005. MR2139233 (2006b:65129)

[12] S.N. Kruzhkov, First order quasi-linear equations in several independent variables, Math. USSR Sbornik, 10: 217-243, 1970.

[13] A. Kurganov and E. Tadmor, New high-resolution central schemes for nonlinear conservation laws and convection-diffusion equations, J. Comp. Phys., 160: 241-282, 2000. MR1756766 (2001d:65135)

[14] H. Nessyahu and E. Tadmor, Non-oscillatory central differencing for hyperbolic conservation laws, J. Comp. Phys., 87: 408-463, 1990. MR1047564(91i:65157)

[15] F. Sabac, The optimal convergence rate of monotone finite difference methods for hyperbolic conservation laws, SIAM J. Numer. Anal., 34: 2306-2318, 1997. MR1480382 (98j:65064) 
[16] C.-W. Shu, Numerical experiments on the accuracy of ENO and modified ENO schemes, J. Comp. Phys., 5: 127-149, 1990.

[17] P. Sweby, High resolution schemes using flux limiters for hyperbolic conservation laws, SIAM J. Numer. Anal., 21: 995-1011, 1984. MR0760628 (85m:65085)

[18] T. Tang and Z.-H. Teng, The sharpness of Kuznetsov's $O(\sqrt{\Delta x}) L^{1}$-errror estimate for monotone difference scheme, Math. Comp., 64: 581-589, 1995. MR1270625 (95f:65176)

Department of Mathematics, Texas A\&M University, College Station, Texas 77845

E-mail address: popov@math.tamu.edu

Department of Mathematics, University of South Carolina, Columbia, South CarOLINA 29208

E-mail address: trifonov@math.sc.edu 